\title{
Do the Herschel cold clouds in the Galactic halo embody its dark matter?
}

\author{
Theo M. Nieuwenhuizen, Erik F. G. van Heusden and \\ Matthew T. P. Liska \\ Institute for Theoretical Physics, University of Amsterdam, Science Park 904, \\ P.O. Box 94485, 1090 GL Amsterdam, The Netherlands \\ email: t.m.nieuwenhuizen@uva.nl
}

\begin{abstract}
Recent Herschel/SPIRE maps of the Small and Large Magellanic Clouds (SMC, LMC) exhibit in each thousands of clouds. Observed at 250 microns, they must be cold, $T \sim 15 \mathrm{~K}$, hence the name "Herschel cold clouds" (HCCs). From the observed rotational velocity profile of the Galaxy and the assumption of spherical symmetry, its mass density is modeled in a form close to that of an isothermal sphere. If the HCCs constitute a certain fraction of it, their angular size distribution has a specified shape. A fit to the data deduced from the SMC/LMC maps supports this and yields $1.7 \mathrm{pc}$ for their average radius. There are so many HCCs that they will make up all the missing Halo mass density if there is spherical symmetry and their average mass is of order $10,000 M_{\odot}$. This compares with the Jeans mass of circa $40,000 M_{\odot}$ and puts forward that the HCCs are in fact Jeans clusters, constituting all the Galactic dark matter and much of its missing baryons, a conclusion deduced before from a different field of the sky (Nieuwenhuizen, Schild and Gibson 2011). A preliminary analysis of the intensities yields that the Jeans clusters themselves may consist of some billion MACHOs of a few dozen Earth masses. With a size of dozens of solar radii, they would mostly not lens but cause occultation of stars in the LMC, SMC and towards the Galactic center, and may thus have been overlooked in microlensing.
\end{abstract}

PACS numbers:

95.35.+d Dark matter (stellar, interstellar, galactic, and cosmological)

98.35.Gi Galactic halo

98.38.Jw Infrared emission

98.56.-p Local group; Magellanic Clouds 


\section{Introduction}

The stone which the builders rejected, the same is become the head of the corner

Matthew, XXI:42

The dark matter riddle was formulated for the Galaxy in 1922 by Jacobus Kapteyn [1]: there must be more mass than in stars, the invisible or dark matter. Currently it is mostly approached from particle searches, aiming to find the weakly interacting massive particles (WIMPs), the purported cold dark matter (CDM) particle [2]. A complementary approach is to investigate statistical properties of dark matter. Since the Galaxy exists billions of years, its dark matter, whatever its nature, probably had time enough to reach some type of equilibrium. This expectation is supported by the observed flattening of galactic rotation curves. After all, a really constant circular rotation velocity $v_{\mathrm{c}}$ would imply, due to the Kepler relation $v_{\mathrm{c}}=[G M(r) / r]^{1 / 2}$, that the mass $M(r)$ inside a sphere of radius $r$ grows linearly in $r$, and hence that the mass density decays as $r^{-2}$. But that is the well known case of the singular isothermal sphere, a solution to the Poisson-Boltzmann equation, where the mass density is a Boltzmann distribution, the equilibrium distribution of standard statistical physics. The Galaxy is close to that.

The question "Which type of dark matter causes the isothermal profile?" has no established solution at present. But galaxy-scale difficulties for WIMP dark matter are abundant, see, e. g., [3]. It is proper to approach the problem in the opposite direction: identify a candidate, and investigate whether it satisfies the relevant statistical criteria.

The answer to this may be related to the missing baryon problem: of all baryons expected from the standard model, $10 \%$ are known to be in stars and $20-30 \%$ in gas of galaxies and galaxy clusters, while the rest, 60-70\%, is still unaccounted for [4]. A new component, hot gas in the Galactic halo that weighs over 10 billion solar masses, has been reported recently [5], but it will likely not be enough to solve the full problem.

Our argument starts with the remarkably low $15 \mathrm{~K}$ temperature of clouds all over the sky, known from IRAS observations, and commonly attributed to dust, but see [6]. For lack of suitable instruments, $15 \mathrm{~K}$ objects would have mostly remained in the dark until recently, but by now Planck detects 915 cold molecular cloud core candidates, out of over 15,000 unique sources that litter the sky at the $857 \mathrm{GHz}(350 \mu)$, the largest Planck frequency, see Fig. 6 of [7]. Several of these clouds have a gas mass of 10,000 solar masses $[8,9]$. Towards the Galactic center, at coordinates $|b|<1^{\circ}$ and $\ell=300^{\circ}-330^{\circ}, 1205$ dark clouds are detected by Herschel/Spitzer, which can be seen not only in absorption, but also in emission at $250 \mu$ and $500 \mu$ [10].

One particular $15^{\circ} \times 12^{\circ}$ region on the sky observed already with BOOMERANG in the 1990's and focused on recently by Veneziani et al (2010) [11], exposes dozens of 7-20 K clouds. Their temperature, typical opening angle, two-dimensional number density and emission intensity are explained from gravitational hydrodynamics (GHD) employing an isothermal sphere of Jeans clusters (JCs) in which, next to stars and 


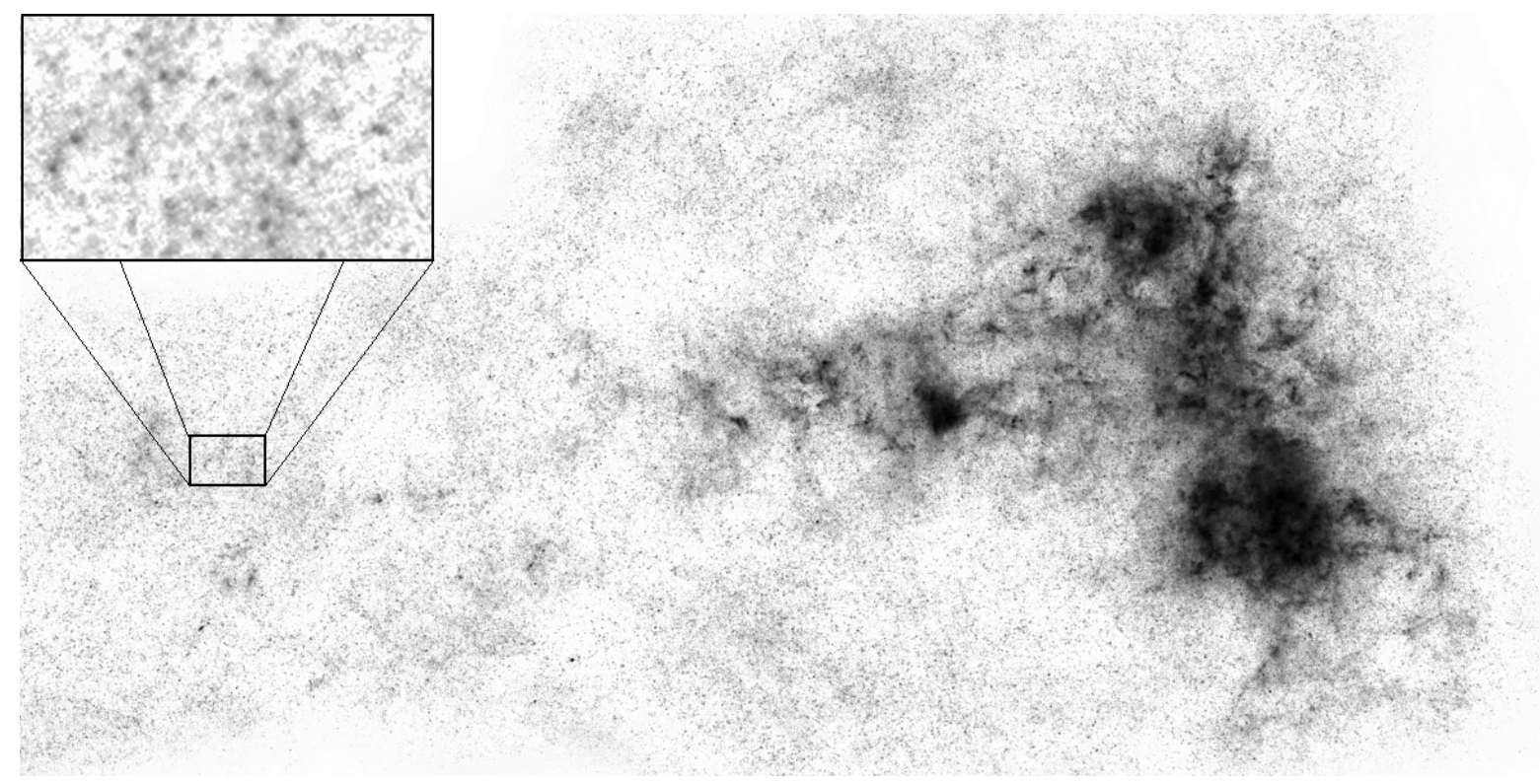

Figure 1. This luminosity-inverted $8.32^{\circ} \times 4.16^{\circ}$ Herschel-SPIRE map of the SMC at $250 \mu \mathrm{m}$ exposes the star-rich bar on the right and the wing towards the left, and thousands of Herschel cold clouds (HCCs) in the Galactic foreground, of different size and intensity. This figure was prepared from data made public by NASA/ESA.

gas, all dynamical mass is contained [12]. It was concluded that these clouds are JCs and make up the galactic dark matter and most of the missing baryons. This challenges the standard $\Lambda \mathrm{CDM}$ or concordance model, and clearly awaits testing by further observations.

Cold $15 \mathrm{~K}$ objects should be clearly exposed by Herschel SPIRE's $250 \mu$ detector. In early 2012 NASA/ESA published a multi-wavelength map of the Small Magellanic Clouds (SMC) [13]. (A similar picture for the Large Magellanic Cloud [14] is less suited for our purpose; it will be discussed in section 5.3.) In the SMC picture one observes the bar on the right and the wing from the right to the middle, which both contain many stars. Our interest is in the $250 \mu$ maps, represented as red dots in these color figures, that we reproduce for the SMC as gray dots in Figure 1. Surprisingly, one observes many hundreds, if not thousands, of HCCs all over the picture (except in regions without data), not exposed before in this number. Since most of them seem absent in the "green" map (160 and $100 \mu \mathrm{m}$ ), they are likely not background galaxies, but must be cold ( $15 \mathrm{~K})$, and we adopt the Juvela (2012) name "Herschel cold clouds" (HCCs). They are the focus of our present study. Our aim is to investigate whether the HCCs can correspond to JCs that would comprise the full dynamic dark matter of the Galaxy [12].

In section 2 we discuss the Jeans theory and its extension by gravitational hydrodynamics, and we recall a number of observations already explained by this model. In section 3 we formulate a model for the expected angular size distribution of the HCCs. In section 4 we investigate their size distribution in the SMC map and in section 5 we 
discuss the fit to our model. In section 6 we discuss the results within the view point of JCs and micro brown dwarfs ( $\mu \mathrm{BDs})$, and we conclude with a discussion.

\section{The Jeans instability and GHD}

The Jeans 1902 mechanism asserts that when in the early Universe the plasma changes into a $\mathrm{H}-\mathrm{He}$ gas, this gas, considered to be infinitely distributed, exhibits instabilities at the Jeans scale, where pressure can no longer overcome the effects of gravitation and diminished heat transfer $[15,16]$. This leads to the formation of Jeans gas clumps that weigh about $40,000 M_{\odot}$. Globular star clusters are supposed to have formed from a handful of coagulated clumps, but other ones are generally considered to have turned into stars, brown dwarfs, planets, gas clouds, etc., so that they should not exist anymore, at least not in large numbers. We shall pose, however, the question: does the majority of the Jeans gas clumps still exist as HCCs?

In GHD it is asserted that, due to turbulence and viscosity, the Jeans clumps themselves fragment in earth mass MACHOs, called primordial gas particles [16] or, what we shall employ here, micro brown dwarfs ( $\mu \mathrm{BDs})[12]$. This turned the Jeans gas clumps into JCs of billions of $\mu \mathrm{BDs}$. In order to reach a temperature of $2.725 \mathrm{~K}$, the $\mu \mathrm{BDs}$ would have to cool from gas to solid, by radiating away the latent heat in order to cross the first-order phase transition line that emerges the $\mathrm{H}$ triple point at $13.8 \mathrm{~K}$. If this takes more than a Hubble time, their temperatures must become stuck somewhere above $14 \mathrm{~K}$.

The wide-binaries problem, the last-parsec problem and the Helium-3 problem also find a simple explanation in GHD [17], as do long-duration radio transients [12, 18, 19]. GHD offers plenty of dark baryons for making new stars even with modest recycling of stellar matter [20,21,22], because it would consider the outflowing and infalling matter as dynamical behavior of the galaxy's large baryonic halo. The $\mu$ BDs would be leaky, so there is gas throughout the JC. This offers an explanation for the Lyman-alpha forest: thousands of extinction lines in quasar spectra due to hydrogen clouds along the sightline. Why these clouds did not dissolve quickly is still a miracle [23], but JCs of leaky $\mu$ BDs provide a simple explanation: the gas is bound by mass concentrations, the $\mu$ BDs. Therefore, the total mass can be much larger than the gas mass, and this is the picture that we shall investigate in the present paper.

The idea can also be tested in our immediate neighborhood. The Local Leo Cold Cloud is a large ( $\sim 5 \mathrm{deg})$, cold $(15-20 \mathrm{~K})$, nearby (10-20 parsec) H cloud [24]. Like the Lyman-alpha clouds, it can be long lived if it contains many unresolved $\mu$ BDs, which bind the gas gravitationally, thus explaining why the gas can have the observed large pressure discussed in [25]. The observed velocity differences inside the Local Leo Cold Cloud are explained by the local winds due to mostly random motion of $\mu$ BDs, that sweep the gas with them.

In GHD, planet and star formation occurs by coalescence of $\mu \mathrm{BDs}[12,26,27]$. They are cold and relatively small now (solar size), but when heated they expand and 
can coagulate. First a gas planet is formed and, after enough steps, a star is created. This explains why galaxy mergers such as Antennae [28] and Tadpole [29] have tails of thousands of young ( $<10 \mathrm{Myr}$ ) globular star clusters, much younger than the event duration of galaxy merging (150-200 Myr): during the merging, the local JCs of one galaxy are heated by the center of the other, so that they later develop stars [12]. Many or possibly all stars may have been formed in such clusters [28].

\subsection{The Jeans mass}

We shall need a mechanism that explains the formation of so many, so regular, so identical cold clouds. A well known candidate is the 1902 Jeans instability of the neutral gas, just as it forms at the recombination. It is often taught as a separate issue in standard courses on astrophysics, but for us it will be a cornerstone.

The Jeans mechanism asserts that at last scattering ( $L$; decoupling, recombination) the plasma turns into a neutral gas, which is unstable on a scale where pressure and heat transport are overcome by gravitation. The free fall time is $\tau_{g}=\left(G \rho_{B}\right)^{-1 / 2}=5.30$ Myr, where the baryonic density at the decoupling at $z_{L}=1088$ is $\rho_{B}=\Omega_{B} \rho_{c}\left(1+z_{L}\right)^{3}=$ $5.3510^{-22} \mathrm{gr} \mathrm{cm}^{-3}$ for $\Omega_{B}=0.045$ and $\rho_{c}=9.2110^{-30} \mathrm{gr} \mathrm{cm}^{-3}$ at $h_{70}=1$. The speed of sound of a monoatomic gas is $v_{s}=(5 p / 3 \rho)^{1 / 2}$. For the gas of $\mathrm{H}$ and $25 \%$ of weight in He the pressure $p=0.813 \rho k_{B} T / m_{N}$ yields $v_{s}=5.68 \mathrm{~km} \mathrm{~s}^{-1}$. The gas fragments at the Jeans scale $L_{\text {Jeans }}=v_{s} \tau_{g}=31$ pc into Jeans clumps with mass

$$
M_{\text {Jeans }}=\frac{\pi}{6} \rho_{B} L_{\text {Jeans }}^{3}=\frac{\pi}{6} \Omega_{B} \rho_{c}\left(1+z_{L}\right)^{3} L_{\text {Jeans }}^{3}=\frac{\pi v_{s}^{3}}{6 G^{3 / 2} \rho_{B}^{1 / 2}}=40,000 M_{\odot} .
$$

A practical way to check this is to consider globular star clusters, which, we presume, are the result of up to a dozen or so of JC mergers, as is exhibited by their sets of stars of different ages [30]. To yield a GC mass between ten thousand and a few hundred thousand solar masses, the Jeans mass should indeed be a few ten thousand solar masses.

\subsection{Nearly isothermal mass density profile}

The circular rotation speed at the position of the Sun, taken as $v_{c}^{*}=220 \mathrm{~km} \mathrm{~s}^{-1}$, drops within $10 \%$ accuracy mildly to $175 \mathrm{~km} \mathrm{~s}^{-1}$ at $60 \mathrm{kpc}$ [31]. This range can be modeled as $v_{c}(r)=v_{c}^{*}\left(d_{\mathrm{SA}} / r\right)^{\varepsilon} \mathrm{km} \mathrm{s}^{-1}$ with $\varepsilon=0.114$ and $d_{\mathrm{SA}}=8.0 \pm 0.5 \mathrm{kpc}$, the distance to SagA* Now it is found that $v_{c}^{*}=240 \pm 14 \mathrm{~km} \mathrm{~s}^{-1}$ [32]. In the presence of spherical symmetry, Kepler's law $G M(r) r^{-1}=v_{c}^{2}(r)$ then yields for the mass density $\rho=M^{\prime}(r) / 4 \pi r^{2}$ :

$$
\rho(r)=\frac{(1-2 \varepsilon) v_{c}^{* 2}}{4 \pi G r^{2}}\left(\frac{d_{\mathrm{SA}}}{r}\right)^{2 \varepsilon}, \quad v_{c}^{*}=240 \pm 14 \mathrm{~km} \mathrm{~s}^{-1} .
$$

For $\varepsilon=0$ this is the singular isothermal sphere model with velocity dispersion $v_{c}^{*} / \sqrt{2}$. 


\section{Model for the angular size distribution of Galactic foregrounds}

We assume that the HCCs make up a fixed fraction $c_{\mathrm{hcc}} \leq 1$ of the total mass density. With average size $\bar{R}$ and average mass $\bar{M}$, this leads us to their average number density

$$
n(r)=\frac{c_{\mathrm{hcc}}}{\bar{M}} \rho(r) \equiv \frac{A}{\bar{R} r^{2}}\left(\frac{d_{\mathrm{SA}}}{r}\right)^{2 \varepsilon}, \quad A=\frac{(1-2 \varepsilon) \bar{R} v_{c}^{* 2} c_{\mathrm{hcc}}}{4 \pi G \bar{M}} .
$$

For the distribution of radii $R$ at position $\mathbf{r}$ we consider the factorized form $n(r, R)=$ $n(r) f(R / \bar{R}) / \bar{R}$ with $\int_{0}^{\infty} \mathrm{d} x f(x)=\int_{0}^{\infty} \mathrm{d} x x f(x)=1$, which reproduces the local average $\int \mathrm{d} R n(r, R)=n(r)$, while evidently $\langle R\rangle=\bar{R}$. We specify to the $\Gamma$-distributions $f(x)=$ $(n x)^{n} \exp (-n x) / x \Gamma(n)$, which for $n \rightarrow \infty$ lead to the fixed- $R$ case $f(x)=\delta(x-1)$.

\subsection{Seen from $S a g A^{*}$}

From SagA*, an HCC of physical radius $R$ is seen with angular radius $\theta=\arctan R / r \approx$ $R / r$ and angular diameter $2 \theta$. Let $N(\theta)$ denote the number of HCCs that have an angular radius smaller than $\theta$. Its differential increase per unit angular radius per unit solid angle is

$$
\frac{\mathrm{d} N(\theta)}{\mathrm{d} \theta \mathrm{d} \Omega}=\frac{1}{\Delta \Omega} \iint \mathrm{d} r \mathrm{~d} \Omega \mathrm{d} R r^{2} n(r, R) \delta\left(\theta-\frac{R}{r}\right),
$$

where $\mathrm{d} \Omega$ is the differential solid angle, the integral of which cancels the factor $\Delta \Omega$. Using $\delta\left(\theta-R r^{-1}\right)=R \theta^{-2} \delta\left(r-R \theta^{-1}\right)$ and defining $x=R / \bar{R}$, the result takes the form

$$
\frac{\mathrm{d} N(\theta)}{\mathrm{d} \theta \mathrm{d} \Omega}=\frac{A C(\theta)}{\theta^{2}}, \quad C(\theta)=\int_{0}^{\infty} \mathrm{d} x f(x) x\left(\frac{d_{\mathrm{SA}} \theta}{x \bar{R}}\right)^{2 \varepsilon}=\frac{\Gamma(n+1-2 \varepsilon)}{\Gamma(n+1)}\left(\frac{n d_{\mathrm{SA}} \theta}{\bar{R}}\right)^{2 \varepsilon},
$$

which has dimension $\operatorname{rad}^{-3}$. Notice that $A$ and $\bar{R}$ do not occur independently here. For $\varepsilon \rightarrow 0$ one considers in fact isothermally distributed HCCs, where this relation has $C=1$ and expresses the isothermal relation $\rho(r) \sim r^{-2}$ in a dimensionless form.

\subsection{Seen from the earth}

The fact that the Sun is not located in the center of the Galaxy introduces a slight complication. But actually it turns out as a benefit that allows us to estimate both the amplitude $A$ and the average radius $\bar{R}$ from the statistics of the data.

A sphere of radius $R=x \bar{R}$ at distance $d$ from the Earth at galactic coordinates $(b, \ell)$ has position $\mathbf{r}=\left(d \cos b \cos \ell+d_{\mathrm{SA}}, d \cos b \sin \ell, d \sin b\right)$ with respect to the center of the Galaxy. The modulus is $r(d, b, \ell)=\left(d^{2}+2 d_{\mathrm{SA}} d \cos b \cos \ell+d_{\mathrm{SA}}^{2}\right)^{1 / 2}$ and the volume element $\mathrm{d}^{3} r=\mathrm{d} d \mathrm{~d} \Omega d^{2}$, where $\mathrm{d} \Omega=\mathrm{d} b \mathrm{~d} \ell \cos b$. Because $\theta=R / d$ we have the definition

$$
\frac{\mathrm{d} N(\theta)}{\mathrm{d} \theta \mathrm{d} \Omega}=\frac{1}{\Delta \Omega} \iint \mathrm{d} d \mathrm{~d} \Omega \mathrm{d} x d^{2} n(r) f(x) \delta\left(\theta-\frac{x \bar{R}}{d}\right)
$$


When $\Delta \Omega$ is a small field around $(\bar{b}, \bar{\ell})$, the integrand hardly varies with $\Omega$, so $\Delta \Omega$ drops out again. The $d$-integral yields $\mathrm{d} N(\theta) / \mathrm{d} \theta \mathrm{d} \Omega=A \theta^{-2} C(\theta)$, with $A$ from (3) and now

$$
C(\theta)=\int_{0}^{\infty} \mathrm{d} x f(x) x \frac{\left(x \bar{R} \theta^{-1}\right)^{2} d_{\mathrm{SA}}^{2 \varepsilon}}{\left[r\left(x \bar{R} \theta^{-1}, \bar{b}, \bar{\ell}\right)\right]^{2+2 \varepsilon}}
$$

\section{Data analysis of Herschel maps of the Magellanic Clouds}

\subsection{Detection}

The Herschel "Spectral and Photometric Imaging Receiver" (SPIRE) instrument has detectors for central wavelengths of $250 \mu, 360 \mu$ and $520 \mu$. The full-width at halfmaximum of the $250 \mu$ detector is $18.1^{\prime \prime}$, see section 6.4 of Ref. [33]. Herschel-SPIRE has delivered a multi-frequency map of the SMC [13]. The $250 \mu$ map has a resolution of $6000 \times 3000$ pixels and its angular size is determined by identifying a number of spots with stars known from catalogs. We thus obtain $8.315^{\circ} \times 4.157^{\circ}$, which corresponds to pixels of linear size of $\theta_{\text {pix }}=4.99^{\prime \prime}$. Hence the resolution limit is 3.6 pixels across.

We shall present data for structures from 8 to 124 pixels in size. Each pixel has a solid angle $\theta_{\text {pix }}^{2}$. Approximating an object with a number of pixels $N_{\text {pix }}$ as a sphere, we define the angular radius $\theta$ by $N_{\text {pix }} \theta_{\text {pix }}^{2}=\pi \theta^{2}$. In the analysis the angular widths are in the range $15^{\prime \prime}<2 \theta<63^{\prime \prime}$, the smallest value being just below the resolution limit, but not causing a clear deviation in Fig. 2.

\subsection{On ImageJ}

To count the HCC's we use the the freely available program "ImageJ". We first split the colors of the multi-frequency map to extract the $250 \mu$ map represented in Fig. 1. Next we mention some relevant points/decisions made with respect to the counting method.

1) First of all, we set the minimum threshold value to 11 and the maximum to 255 (on a 256 bit gray-scale) to select the pixels with a grayscale between 11 and 255. If the minimum threshold value is set much higher, visible absence of data will occur; setting the threshold much lower than 11 causes too much background noise. Here we define background noise as very faint objects measuring less than $2 \times 2$ pixels, which is below the diffraction limit of $\sim 4 \times 4$ pixels for Herschel's 3.5 meter primary mirror at this wavelength.

2) We use a watershed function to split overlapping HCCs. This also helps the edge tracker (see point 5) to distinguish between the background and HCCs.

3) We count the number $\Delta N$ of HCCs for pixel sizes between 6 and 125 .

4) Large distortions (defined as HCCs bigger than $~ 130$ pixels), like luminous matter clumps, are unusable. They occupy $45-55 \%$ of the map, while some $12 \%$ of the map is without any data. In such regions we normalize by the analyzed area, see 6).

5) For the counting we use an edge tracker to help distinguishing nearby/overlapping HCCs. However, it seems that the edge tracker increases the apparent area size of HCCs 
as a function of their true area size. We correct for this issue in our data by the empirical relation $N_{\text {pix }}^{\text {true }}=(0.11 \pm 0.04)\left(N_{\text {pix }}^{\text {apparent }}\right)^{1.41 \pm 0.01}$.

6) We bin the data in sets of $\Delta N_{\text {pix }}=4$ pixels, viz. $N_{\text {pix }}=(6,7,8,9),(10,11,12,13)$, etc., with central bin values $\bar{N}_{\text {pix }}=7.5,11.5$ etc., to be used in the definition of $\theta$. We determine $\theta^{2} \mathrm{~d} N / \mathrm{d} \theta \mathrm{d} \Omega \equiv 2 \pi^{-1 / 2} \bar{N}_{\text {pix }}^{3 / 2} \theta_{\text {pix }} \cdot \Delta N / \Delta N_{\text {pix }} \Delta \Omega$, where $\Delta N$ is the number of HCCs in the bin of the investigated region of angular area $\Delta \Omega=\cos \bar{b} \Delta b \Delta \ell$.

7) We determine the error bars by measuring 7 different areas in our data set and calculating the standard deviation for each bin of 4 pixels.

\subsection{Two ways of analysis for the $S M C$}

We divide the SMC map into 7 "good" regions that are poor in stars and define region 8 as the rest, centering on the star-rich regions, on the border regions without data, and on other regions with poor data. The average angular position is $(\bar{b}, \bar{\ell})=\left(-44.2^{\circ}, 302.8^{\circ}\right)$.

In our first method, we analyze the full SMC map. ImageJ treats the luminous regions as big HCCs of 10,000 pixels and more, so they do not add to our counts. We correct the useful area by subtracting these big luminous regions, as well as regions of HCCs with more than 130 pixels. We do the same for the side regions without data. The error in the variable $y \equiv \theta^{2} \mathrm{~d} N(\theta) / \mathrm{d} \theta \mathrm{d} \Omega$ is defined as $\Delta y=\left[\sum_{i=1}^{n} w_{i}\left(y_{i}-\bar{y}\right)^{2} \sum_{i=1}^{n} w_{i}^{2}\right]^{1 / 2}$, where $n=8$ and $w_{i}=\Delta \Omega_{i} / \sum_{j=1}^{n} \Delta \Omega_{j}$ is the areal weight of the region $i$ and $\bar{y}=\sum w_{i} y_{i}$.

In our second method for the SMC, we select the seven "good" areas away from the star-rich regions, we analyze them and we join them by area size. The error in the variable $y$ then sums over these $n=7$ areas.

For the LMC only a strip on the left and a smaller strip on the right are useful for our analysis. The errors are now larger, since we only have two regions $(n=2)$ with relatively few HCCs. The average position of the LMC is $(\bar{b}, \bar{\ell})=\left(-32.89^{\circ}, 280.47^{\circ}\right)$.

\subsection{Provisos of our method}

We take for granted that the "red dots" of the color version of the SMC map (the gray ones in Fig. 1) represent physical objects and we model them as spheres. One may argue that the observations present more edgy or hairy objects, which could be an effect of a mutual interactions, near passages or central collisions, or complete absence of nearly spherical objects. But most of these issues concern details below the resolution limit, which cannot be analyzed properly. In the case of large objects, we assume that they are agglomerations of spheres, and separate them accordingly. But there can be mass streams between them, in particular due to mutual interactions. However, given the size of our statistical errors, these issues do not seem too relevant at our level of description.

The LMC map [14] is largely dominated by the star-rich region. For our analysis small side regions remain available, the results of which agree with those for the SMC.

Although we consider our model as reasonable and the parameters come out as reasonable, we stress that the data could be fit to many other models.

We have analyzed only the MC regions, which cover a small piece of the sky. 


\begin{tabular}{|l|l|r|r|l|l|r|r|r|}
\hline \hline Region & $n$ & $\chi^{2}$ & $\chi^{2} / \nu$ & $A$ & $\bar{R}(\mathrm{pc})$ & $\left.\bar{\theta}_{\mathrm{MC}}{ }^{\prime \prime}\right)$ & $\bar{M}\left(10^{3} M_{\odot}\right)$ & $\sigma_{\mu \mathrm{bd}}(\mathrm{km} / \mathrm{s})$ \\
\hline \hline SMC-total & $\infty$ & 23.6 & 0.84 & $102 \pm 4$ & $1.68 \pm 0.18$ & $5.7 \pm 0.6$ & $13.5 \pm 1.9$ & $4.16 \pm 0.07$ \\
\hline SMC-good & $\infty$ & 67.1 & 2.40 & $106 \pm 3$ & $1.82 \pm 0.18$ & $6.2 \pm 0.6$ & $14.1 \pm 1.8$ & $4.08 \pm 0.06$ \\
\hline LMC-sides & $\infty$ & 12.5 & 0.45 & $113 \pm 14$ & $1.17 \pm 0.19$ & $5.0 \pm 0.8$ & $8.6 \pm 2.4$ & $3.97 \pm 0.25$ \\
\hline S+L MC & $\infty$ & 38.1 & 0.66 & $104 \pm 3$ & $1.65 \pm 0.15$ & $5.6 \pm 0.5$ & $13.1 \pm 1.6$ & $4.14 \pm 0.07$ \\
\hline \hline SMC-total & 2 & 22.4 & 0.80 & $110 \pm 5$ & $1.30 \pm 0.18$ & $4.4 \pm 0.6$ & $9.8 \pm 1.7$ & $4.02 \pm 0.08$ \\
\hline SMC-good & 2 & 65.5 & 2.34 & $114 \pm 4$ & $1.43 \pm 0.18$ & $4.8 \pm 0.6$ & $10.4 \pm 1.7$ & $3.95 \pm 0.07$ \\
\hline LMC-sides & 2 & 13.4 & 0.48 & $127 \pm 20$ & $0.79 \pm 0.18$ & $3.4 \pm 0.8$ & $5.2 \pm 1.9$ & $3.74 \pm 0.29$ \\
\hline S+L MC & 2 & 37.5 & 0.65 & $112 \pm 4$ & $1.25 \pm 0.14$ & $4.2 \pm 0.5$ & $9.2 \pm 1.4$ & $3.98 \pm 0.08$ \\
\hline \hline
\end{tabular}

Table 1. Fits of the model for Jeans clusters for the four data sets: the total SMC, the good regions of the SMC, the sides of the LMC, and the total SMC combined with the sides of the LMC. $A$ is an amplitude; $\bar{R}$ the average radius of the HCC; $\bar{\theta}_{\mathrm{MC}}$ refers to $\bar{\theta}_{\mathrm{SMC}}$, the angular radius of an average $\mathrm{HCC}$ located at the SMC, except for the LMC-sides cases, where it correspondingly refers to the LMC. Finally, $\bar{M}$ is the average HCC mass needed to explain all dynamical mass in the Galaxy, and $\sigma_{\mu \text { bd }}$ is the velocity dispersion of the $\mu$ BDs. The upper data refer to HCCs with a fixed radius $(n=\infty)$ and the lower to radii taken from the $n=2 \Gamma$-distribution $f(x)=4 x \exp (-2 x)$.

In this study we did not have access to the infrared intensities, which give additional information about the ratio of their radiating surface to their distance squared.

\section{Fits to the nearly isothermal model}

\subsection{Towards the $S M C$, the case of equal radii}

A $\chi^{2}$ fit of the theory of previous section to the observed 30 binned values of $\theta^{2} \mathrm{~d} N / \mathrm{d} \theta \mathrm{d} \Omega$ has been performed, see Fig. 2. For the full SMC picture the fit yields

$$
A=102 \pm 4, \quad \bar{R}=1.68 \pm 0.18 \mathrm{pc}, \quad \chi^{2}=23.6, \quad \frac{\chi^{2}}{\nu}=0.84
$$

where $\nu=30-2$ is the number of degrees of freedom. The error bars derive from the inverse correlation matrix of the fit parameters $p_{1} \equiv A$ and $p_{2} \equiv \bar{R} \mathrm{pc}^{-1}$ for the prediction $y_{i} \equiv A C\left(\theta_{i} ; \bar{R}\right)$, viz. $\quad C_{a b}^{-1}=2 \sum_{i}\left(\partial y_{i} / \partial p_{a}\right)\left(\partial y_{i} / \partial p_{b}\right) \sigma_{i}^{-2}$. It follows that $\left\langle\delta A^{2}\right\rangle \equiv C_{11}=22.0,\langle\delta A \delta \bar{R}\rangle \mathrm{pc}^{-1} \equiv C_{12}=-0.71$ and $\left\langle\delta \bar{R}^{2}\right\rangle \mathrm{pc}^{-2} \equiv C_{22}=0.032$.

The angular radius of a HCC of average size, located in the SMC, is $\bar{\theta}_{\mathrm{SMC}}=\bar{R} / d_{\mathrm{SMC}}$, with $d_{\mathrm{SMC}}=61 \pm 3 \mathrm{kpc}$ the SMC distance. Hence $\bar{\theta}_{\mathrm{SMC}}=(5.7 \pm 0.6)^{\prime \prime}$, which is indicated in Fig. 2. (By linear regression, we express here and below all errors in terms of $C_{a b}$.)

To test whether the HCCs can belong mainly to the SMC, we follow our second method and discard the data from the star-rich SMC region, about $51 \%$ of all data. But this appears to yield only minor shifts in $A$ and $\bar{R}$, see the second row in Table 1. 


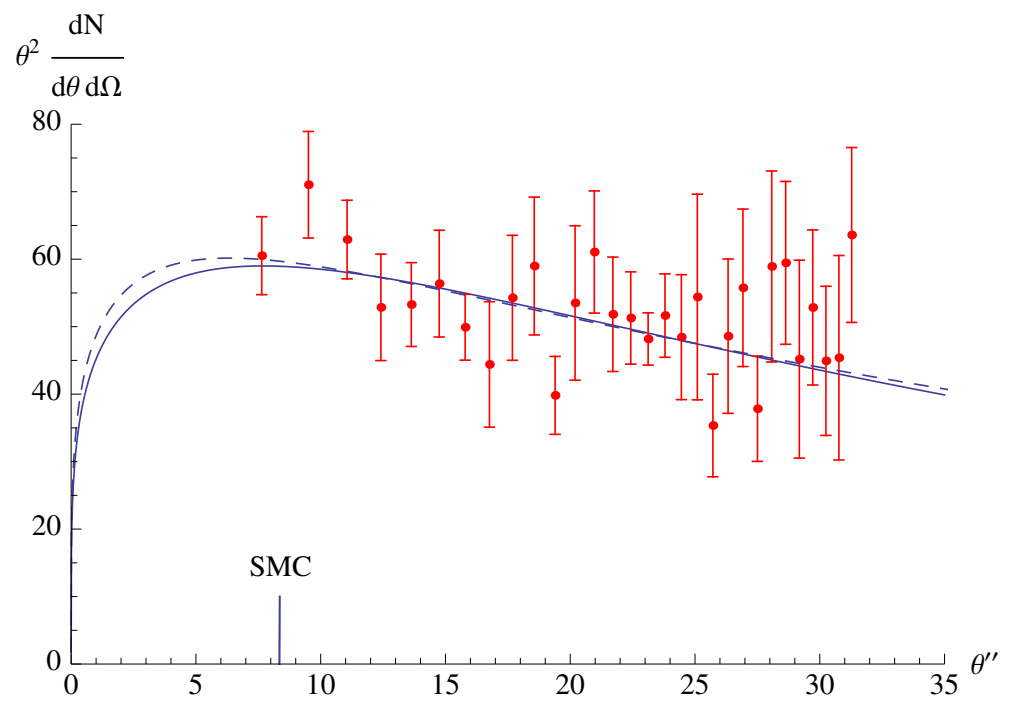

Figure 2. $N(\theta)$ is the number of Herschel cold clouds (HCCs) with angular radius smaller than $\theta$. The data for $\theta^{2} \mathrm{~d} N / \mathrm{d} \theta \mathrm{d} \Omega\left(\right.$ in $\operatorname{rad}^{-1}$ ) as function of $\theta$ (in arcsec) are deduced from Fig. 1. The full curve is the best fit for HCCs of equal radius and the dashed curve for HCC radii taken from a $\Gamma$-distribution with $n=2$. The initial growth stems from the $\theta^{2 \varepsilon}$ factor and the decay from the power of $d r^{-1}$ in Eq. (7). The vertical bar denotes the angular radius $5.7^{\prime \prime}$ of an HCC of average radius located in the SMC.

Both the small values of $\bar{\theta}_{\mathrm{SMC}}$ and the minor shifts with respect to leaving out star-rich regions reject the assumption that the HCCs of Fig. 1 belong to the SMC. Hence we conclude that they nearly all belong to the Galactic Halo.

The investigated distances stem from $\bar{R} / \theta$ and lie between 10 and $40 \mathrm{kpc}$. All fit parameters are tabulated in Table 1 . As a canonical average radius, we adopt $\bar{R}=2$ pc.

\subsection{Towards the $S M C$, spread in radii}

The radii of the HCCs can be random, mixing angular sizes of large, far HCCs with nearby, small ones. As an example, we consider the $n=2 \Gamma$-distribution $f(x)=$ $4 x \exp (-2 x)$. Rows 5,6 and 8 in Table 1 show differences with respect to the equal- $R$ cases (rows 1,2 and 4, respectively). These modest deviations make it unlikely that the size distribution can play an important role in the physical interpretation of the results.

\subsection{Towards the $L M C$}

A similar map exists for the LMC [14] and in the data acquisition, described above, we only use two side regions. Now the error bars are much larger, which actually leads to a small $\chi^{2}$ (Table 1, rows 3 and 7 ). We consider the deviations from the fit parameters for the SMC (Table 1, rows 1, 2 and 5,6) to be statistically unimportant. 


\subsection{SMC combined with $L M C$}

Combining the 30 data for the total SMC with the 30 for the LMC, hardly changes the parameters of the total SMC alone and decreases only slightly the error bars (Compare rows 5 and 8 in table 1 with rows 1 and 4, respectively). This shows again that the LMC data, with their larger error bars, have low statistical weight.

\section{Interpretation in terms of Jeans clusters and micro brown dwarfs}

Recalling Eq. (3), we get for the average mass of the HCCs from the SMC

$$
\frac{\bar{M}}{c_{\mathrm{hcc}}}=\frac{\bar{R} v_{c}^{* 2}(1-2 \varepsilon)}{4 \pi G A}=(13.5 \pm 1.9) 10^{3} M_{\odot} \text { or }(14.1 \pm 1.8) 10^{3} M_{\odot} .
$$

The first (second) estimate holds the assumption of fixed radius for the full (restricted) data set; for the case of random $R$ this becomes $(9.8 \pm 1.7) 10^{3} M_{\odot}$ or $(10.4 \pm 1.7) \times 10^{3} M_{\odot}$ for these sets, respectively. For the LMC the results deviate, see Table 1, but, as discussed, they have lower statistical weight.

Without further input, $c_{\text {hcc }}$ may take a value of order $10^{-3}-10^{-4}$, the case of solar

mass gas clouds, as often concluded from gas masses alone. However, if the clouds are composed of leaky $\mu \mathrm{BD}$ s, the total mass can be much larger.

The Jeans mass has been estimated as $40,000 M_{\odot}$ in Eq. (1). Therefore, it is imperative to discuss the case $c_{\mathrm{hcc}}=1$, for which the HCCs represent the complete dynamical dark matter of the Galaxy. One then identifies the HCCs of heretofore unknown origin with the JCs predicted by the Jeans instability and the GHD fragmentation into $\mu$ BDs. In view of table 1, we adopt the canonical average mass $\bar{M}_{\mathrm{jc}}=10,000 M_{\odot}$. This lies within the range of HCC masses deduced from gas alone $[8,9,35]$. The typical distance to the nearest $\mathrm{JC}$ is $d_{1}=\left[\rho\left(d_{\mathrm{SA}}\right) / \bar{M}_{\mathrm{jc}}\right]^{-1 / 3}=92 \mathrm{pc}$, its angular radius being $1.1^{\circ}$. Cold structures of size $\sim 0.5^{\circ}$ have been discussed in [11], while the HCCs analyzed in [8, 9] have distances between $225 \mathrm{pc}$ and $1.67 \mathrm{kpc}$.

If the JCs themselves are isothermal distributions of $\mu$ BDs with velocity dispersion $\sigma_{\mu \mathrm{bd}}$, their mass and radius are related as $G M_{\mathrm{jc}}=2 \sigma_{\mu \mathrm{bd}}^{2} R_{\mathrm{jc}}$. Assuming that $\sigma_{\mu \mathrm{bd}}$ is basically a constant, this relation also holds for the average. We can thus express $A$ from (3) with $c_{\mathrm{hcc}}=1$ as $A=(1-2 \varepsilon) v_{c}^{* 2} / 8 \pi \sigma_{\mu \mathrm{bd}}^{2}$, which yields, for fixed- $R, \sigma_{\mu \mathrm{bd}}=4.16 \pm 0.07$ $\mathrm{km} \mathrm{s}^{-1}\left(4.08 \pm 0.06 \mathrm{~km} \mathrm{~s}^{-1}\right)$ for the first (second, restricted) SMC data set, and when modeled with random radii $\sigma_{\mu \mathrm{bd}}=4.02 \pm 0.08 \mathrm{~km} \mathrm{~s}^{-1}\left(3.95 \pm 0.07 \mathrm{~km} \mathrm{~s}^{-1}\right)$, respectively. The LMC yields $3.97 \pm 0.25 \mathrm{~km} \mathrm{~s}^{-1}$ for fixed $R$ and $3.74 \pm 0.29 \mathrm{~km} \mathrm{~s}^{-1}$ for random $R$. As canonical value we adopt the value $\sigma_{\mu \mathrm{bd}}=4 \mathrm{~km} \mathrm{~s}^{-1}$, known from stars in globular clusters. The value $c_{\mathrm{hcc}}=1$ is also desired to avoid a too small $\sigma_{\mu \mathrm{bd}} \approx 4 c_{\mathrm{hcc}}^{1 / 2} \mathrm{~km} \mathrm{~s}^{-1}$.

\subsection{On intensities}

At the time of this research, the intensities of the SMC map were not made public, so they were not considered. By now the rough data are available on the Herschel site and 
clean data are to be produced from them (M. Meixner, private discussion). In a future study we intend to incorporate that information for a further test of our model.

Here we look at a few characteristics. The Planck function gives the amount of electromagnetic energy radiated by a black body in thermal equilibrium per unit of time, frequency, area and solid angle, $B_{\nu}(T)=2 h \nu^{3} c^{-2}\left[\exp \left(h \nu / k_{B} T\right)-1\right]^{-1}$. If the $\mu \mathrm{BD}$ surface is optically thick, one expects a nearly exact Planck spectrum. The $\mu \mathrm{BDs}$ in a given JC may have a distribution $\tau(T)$ of temperatures, which leads to $\bar{B}_{\nu}=$ $\int \mathrm{d} T \tau(T) B_{\nu}(T)$. In the modeling as cosmic dust, one often takes $\bar{B}_{\nu} \approx\left(\nu / \nu_{0}\right)^{\beta} B_{\nu}\left(T_{0}\right)$ with spectral index $\beta \approx 2$ [8]. Here we continue with assuming a pure Planck spectrum.

The radiation into the outward hemisphere picks up an angular factor $\pi$. The energy emitted per second per $\mathrm{Hz}$ by $N_{\mu \mathrm{bd}}$ thermal spheres of radius $R_{\mu \mathrm{bd}}$ is $4 \pi^{2} N_{\mu \mathrm{bd}} B_{\nu} R_{\mu \mathrm{bd}}^{2}$. Measured per unit area at distance $d$, this amounts to a specific intensity

$$
I_{\nu}=\pi B_{\nu} \frac{N_{\mu \mathrm{bd}} R_{\mu \mathrm{bd}}^{2}}{d^{2}}=\pi B_{\nu} \frac{M_{\mathrm{jc}} R_{\mu \mathrm{bd}}^{2}}{M_{\mu \mathrm{bd}} d^{2}}=\pi B_{\nu} \frac{\sigma_{\mu \mathrm{bd}}^{2} R_{\mathrm{jc}} R_{\mu \mathrm{bd}}}{\sigma_{\mathrm{gas}}^{2} d^{2}}=\pi B_{\nu} \frac{\sigma_{\mu \mathrm{bd}}^{2} R_{\mu \mathrm{bd}}}{\sigma_{\mathrm{gas}}^{2} R_{\mathrm{jc}}} \frac{\theta^{2}}{\mathrm{sr}} .
$$

In the last identities we model, next to the JCs, also the $\mu \mathrm{BDs}$ as isothermal spheres [12]. They are supposed to consist of gas, $\mathrm{H}$ with the primordial He fraction $\phi=25 \%$ in weight. Neglecting metals, this leaves $\rho_{\mathrm{H}}=n_{\mathrm{H}} m_{N}=(1-\phi) \rho$. With $p=\left(n_{\mathrm{H}}+n_{\mathrm{He}}\right) k_{B} T$, we denote $\sigma_{\text {gas }}^{2} \equiv p / \rho=\left(1-\frac{3}{4} \phi\right) k_{B} T / m_{N}$, which yields $\sigma_{\text {gas }}=317 \mathrm{~m} \mathrm{~s}^{-1}(T / 15 \mathrm{~K})^{1 / 2}$.

In a statistical model we may define the intrinsic JC intensity $i_{\nu} \equiv I_{\nu} d^{2} R^{-2}=I_{\nu} \theta^{-2}$, and assume local fluctuations with density $f_{\nu}\left(x, i_{\nu}\right)$ normalized as $\int \mathrm{d} i f_{\nu}(x, i)=f(x)$. This yields $\mathrm{d} N / \mathrm{d} \theta \mathrm{d} \Omega \mathrm{d} I_{\nu}=A \theta^{-4} C_{\nu}\left(\theta, I_{\nu} \theta^{-2}\right)$ with $C_{\nu}(\theta, i)$ from $(7)$ with $f(x) \rightarrow f_{\nu}(x, i)$.

In the raw $250 \mu$ Herschel data each pixel has size $\theta_{\text {pix }}=6^{\prime \prime}$ and a saturation value between 0 and a few, in units of $\mathrm{Jy} \mathrm{beam}^{-1}$. We denote by $s_{\mathrm{jc}}$ the average saturation value of the pixels that expose the considered JC. The effective beam area is $\Omega_{\text {beam }}=9.2810^{-9} \mathrm{sr}$ [34], so the intensity recorded by a pixel with saturation $s_{\mathrm{jc}}$ is $91.2 s_{\mathrm{jc}}$ mJy. A JC of angular radius $\theta$ involves a number of pixels $N_{\text {pix }}=\pi \theta^{2} \theta_{\text {pix }}^{-2}$ and an intensity $I_{\nu}=s_{\mathrm{jc}} \pi \theta^{2} \Omega_{\text {beam }}^{-1} \mathrm{Jy}$. Equating this with (10) yields an estimate for the $\mu \mathrm{BD}$ radius,

$$
R_{\mu \mathrm{bd}}=\frac{s_{\mathrm{jc}} \mathrm{sr}}{\Omega_{\mathrm{beam}}} \frac{\mathrm{Jy}_{\mathrm{y}}}{B_{\nu}} \frac{\sigma_{\mathrm{gas}}^{2}}{\sigma_{\mu \mathrm{bd}}^{2}} R_{\mathrm{jc}}=53.6 R_{\odot} \frac{s_{\mathrm{jc}}}{0.05} \frac{T}{15 \mathrm{~K}} \frac{B_{\nu}(15 \mathrm{~K})}{B_{\nu}(T)}\left(\frac{4 \mathrm{~km} \mathrm{~s}^{-1}}{\sigma_{\mu \mathrm{bd}}}\right)^{2} \frac{R_{\mathrm{jc}}}{2 \mathrm{pc}},
$$

i.e., dozens of solar radii. The $\mu \mathrm{BD}$ mass then follows as a good dozen of Earth masses,

$$
M_{\mu \mathrm{bd}}=18.8 M_{\oplus} \frac{s_{\mathrm{jc}}}{0.05}\left(\frac{T}{15 \mathrm{~K}}\right)^{2} \frac{B_{\nu}(15 \mathrm{~K})}{B_{\nu}(T)}\left(\frac{4 \mathrm{~km} \mathrm{~s}^{-1}}{\sigma_{\mu \mathrm{bd}}}\right)^{2} \frac{R_{\mathrm{jc}}}{2 \mathrm{pc}} .
$$

With typically $s_{\mathrm{jc}} \sim 0.05$ this compares with the estimate of $13 M_{\oplus}[36]$, and is somewhat larger than the $\sim 3 M_{\oplus}$ deduced from quasar microlensing observation [37].

We can also consider the microlensing of an SMC star by an $\mu \mathrm{BD}$ inside one of the JCs in front of the SMC, at distance $d=x d_{\mathrm{SMC}}$ with $x<1$. The Einstein radius reads: 


$$
R_{E}=\frac{2}{c} \sqrt{x(1-x) G M_{\mu \mathrm{bd}} d_{\mathrm{SMC}}}=0.34 R_{\mu \mathrm{bd}} \frac{\sigma_{\mu \mathrm{bd}}}{4 \mathrm{~km} \mathrm{~s}^{-1}} \sqrt{\frac{4 x(1-x)}{20 s_{\mathrm{jc}}} \frac{B_{\nu}(T)}{B_{\nu}(15 \mathrm{~K})} \frac{2 \mathrm{pc}}{R_{\mathrm{jc}}}} .
$$

(For a star towards the Milky Way the factor 0.34 would be at most 0.12.) Unless $T$ is well above $15 \mathrm{~K}$, one typically has $R_{E}<R_{\mu \mathrm{bd}}$. This is the case of occultation, where the big $\mu \mathrm{BD}$ absorbs the direct light when it passes in front of an SMC star, while its outer atmosphere refracts light [38]. With the $\mu \mathrm{BD}$ speed dominated by that of the respective $\mathrm{JC}$ in which it is embedded, the lensing-occultation event would last $t_{\mathrm{occ}} \sim 2 R_{\mu \mathrm{bd}} / v_{c}^{*} \sim 3.5$ days.

\section{Conclusion}

Early in 2012, an intriguing multifrequency map of the Small Magellanic Cloud was made public. In its color presentation, it contains thousands of red dots, Herschel cold clouds (HCCs), related to the 250 micron observations that we present in Fig. 1.

As a second year project at the University of Amsterdam, it was proposed to analyze the distribution of these HCCs using the program "ImageJ". With only the angular shapes available, no statement can be made about individual objects, so the approach has to embody a statistical analysis (discussed in section 4) and a comparison with theory. Preliminary results were reported at the end of the term [39].

In the theoretical modeling we assume that the Galactic halo is essentially spherically symmetric, taking into account that the dark matter, whatever its nature, is probably well mixed. A statistical analysis is promising, because it exhibits a scaling behavior which, if valid in some region, may apply to the full Halo.

Since we count many (6600) HCCs in the $50 \%$ usable part of the $\sim 8^{\circ} \times 4^{\circ}$ SMC field, we assume that they represent a certain fraction of the total mass, and are well mixed too, so that their distribution has the same shape as the dynamical mass distribution. The Galaxy's slowly decaying circular rotation speed is modeled as $v_{c}(r)=v_{c}^{*}\left(d_{\mathrm{SA}} / r\right)^{\varepsilon}$ with $\varepsilon=0.11$, which corresponds to the nearly isothermal $\left(\sim r^{-2-2 \varepsilon}\right)$ mass density for the Galaxy, and, in the model, to an $r^{-2-2 \varepsilon}$ number density for the HCCs. We allow that the density has also local fluctuations in the HCC size. The number of HCCs per unit angular radius per unit solid angle, $\mathrm{d} N(\theta) / \mathrm{d} \theta \mathrm{d} \Omega$, behaves roughly as $A \theta^{-2}$ for $5^{\prime \prime}<\theta<30^{\prime \prime}$. The amplitude $A \sim 100$ is reasonable, taking into account that typically $\theta \sim 20^{\prime \prime}$. This corresponds to an amount of HCCs in the field of Fig. 1 of the order $8^{\circ} \times 4^{\circ} \times \mathrm{d} N / \mathrm{d} \Omega \sim 0.01 \theta \mathrm{d} N / \mathrm{d} \theta \mathrm{d} \Omega \sim 0.01 A / \theta \sim 10,000$, indeed about twice the number 6600 of HCCs with $N_{\text {pix }} \geq 6$ that we count up to $40 \mathrm{kpc}$ in the usable areas.

Another intuitive interpretation of our numbers is as follows. If we take our SMC count of $6600 \mathrm{HCCs}$, multiply this by 5 for covering the Halo up to $200 \mathrm{kpc}$ and then by $4 \pi /\left(50 \% \times 8.3^{\circ} \times 4.1^{\circ}\right)=2400$ for covering the whole sky, we arrive at an expected

total number of $7.910^{7} \mathrm{HCCs}$ for the Galaxy; if they weigh $10,000 M_{\odot}$ on average, this constitutes $0.7910^{12} M_{\odot}$ in total. This rough estimate stems with the expected 
total Galactic mass, and can be compared with the $1.110^{12} M_{\odot}$ from Eq. (2), that is incorporated in our detailed modeling of the spatial HCC distribution.

Seen from the Earth, nearby objects look larger than from SagA*, which induces a depletion of the HCC histogram towards larger angular radii, up to a factor 2 at $30^{\prime \prime}$. By itself this has little statistical meaning, since our error bars are large. However, our distance to SagA* is big enough to yield a sizable effect in the prediction as well as a good fit for the average HCC radius. The case of equal radii brings $\bar{R}=1.7 \mathrm{pc}$; for comparison, the average half-light radius of 157 GCs in the Milky Way is 4.3 pc [40]; being mostly heavier, GCs probably arise from agglomerates of HCCs. This value of $\bar{R}$ sets the distance scale of Figs. 1 and 2, and relates the investigated angles to distances between 10 and $40 \mathrm{kpc}$. The HCCs bigger than the diffraction limit appear to be located inside the Galactic Halo, in front of the SMC. The less informative LMC map provides results roughly consistent with those for the SMC.

Our SMC-LMC analysis is compatible with a nearly isothermal distribution of Herschel cold clouds. If they are distributed in a spherically symmetric fashion throughout the Halo, they can explain the full dynamical mass of the Galaxy, provided their average mass is of the order of $10,000 M_{\odot}$. One is thus led to identify the HCCs of heretofore unknown origin with the Jeans clusters (JCs) predicted by the Jeans instability. Our mass value is in accord with the Jeans instability, which is known to have produced globular star clusters, probably from mergers of JCs. The value of $10,000 M_{\odot}$ is even somewhat smaller than might be expected, which may be due to an over-concentration of Jeans clusters in front of the Magellanic clouds. Indeed, some clustering, that we observe at small scale, should also be expected on larger scale.

Thousands of similar cold clouds have been detected by Planck [7] and by Herschel/Spitzer [10]. Both the parsec size and 10,000 $M_{\odot}$ weight of the supposed Jeans clusters are in accord with the size and gas masses of some of these clouds $[8,9,10]$.

The amplitude $A$ fixes the ratio $\bar{M}_{\text {jc }} / \bar{R}_{\text {jc }}$, and since in Gravitational Hydrodynamics the JCs themselves are isothermal spheres of micro brown dwarfs ( $\mu$ BDs) of Earth weight scale, it determines their velocity dispersion as $\sigma_{\mu \mathrm{bd}} \approx 4 \mathrm{~km} \mathrm{~s}^{-1}$, a value known from stars in globular star clusters, in support of their presumed connection to Jeans clusters.

Another surprise is that the deduced $\sigma_{\mu \mathrm{bd}}$ is close to the speed of sound at recombination $5.7 \mathrm{~km} \mathrm{~s}^{-1}$, the early moment in the history of the Universe (at 377.000 years after the Big Bang) when the plasma converted into gas. Intriguingly, the $\mu \mathrm{BDs}$ still seem to move at nearly the speed of sound of the gas that fragmented to create them!

Our analysis points at occultation rather than lensing, which may explain why $\mu \mathrm{BDs}$ (MACHOs) can exist but were not observed in microlensing towards the LMC, SMC and the Milky Way [41, 42]: they may not act as lenses but as refractive dark objects. An occultation event in Centaurus A has lasted 170 days [43]. The cloud's mass of $3-10 M_{\oplus}$ agrees with (12); its size of Pluto-orbit scale can be caused by heating of the $\mu \mathrm{BD}[16]$. A documented doubly-peaked quasar microlensing event [44] can be explained as lensing with partial occultation, due to a cloud of about an Earth 
mass and a size of a few solar radii, located in the Halo. This support our present findings, including occultation towards the MCs [45].

We conclude that, under the assumption of a spherically symmetric Halo, enough cold clouds have been observed towards the Magellanic clouds for the Jeans mechanism to explain the full dynamical mass of the Galaxy. In other words, cold JCs can explain the dark matter and most of the missing baryons of the Galaxy. The obvious implication is that CDM would not be needed to explain dynamical behavior in the Galaxy and, by default, at galactic scales. This conclusion was reached before on a the basis of another field of the sky and by other arguments [12]. If CDM does not occur in the Galaxy, then it likely does not exist at all. But this raises the question: What is the true non-baryonic dark matter? A fit to lensing data of the galaxy cluster Abell 1689 has shown that 1.5 $\mathrm{eV}$ neutrinos answer the question [46]. This mass exceeds estimates based on the cold dark matter paradigm, but lies below the upper bound of $2 \mathrm{eV}$. It will be tested in the upcoming KATRIN tritium-decay experiment [47].

As an outlook, the full data for the SMC will also contain the intensities of the HCCs. Investigation of other fields on the sky seems feasible. A dedicated MACHO search towards the LMC can look for refractive lensing-occultation events.

\section{Acknowledgements}

We thank for discussion with Rudy E. Schild, Katerina Dohnalová and Ian Avruch.

\section{References}

[1] J. C. Kapteyn, First attempt at a theory of the arrangement and motion of the Sidereal system, Astrophys. J. 55, 302 (1922).

[2] G. Bertone, D. Hooper and J. Silk, Particle dark matter: evidence, candidates and constraints, Physics Reports 405, 279 (2005).

[3] M. S. Pawlowski, P. Kroupa and K. de Boer, Making Counter-Orbiting Tidal Debris: The Origin of the Milky Way Disc of Satellites, Astron. \& Astrophys. 532, A118 (2011).

[4] F. de Paolis, G. Ingrosso, A.A. Nucita et al., CMB as a possible new tool to study the dark baryons in galaxies, arXiv:1202.2969 (2012).

[5] A. Gupta, S. Mathur, Y. Krongold et al., A huge reservoir of ionized gas around the Milky Way: account for the missing mass? Astrophys. J. 756, L8 (2012).

[6] R. E. Schild, C. H. Gibson, Th. M. Nieuwenhuizen and N. Chandra Wickramasinghe, Why don't clumps of cirrus dust gravitationally collapse?, Physica Script. T151, 014084 (2012).

[7] Planck Collaboration: P. A. R. Ade, N. Aghanim, M. Arnaud et al., Planck early results. VII. The Early Release Compact Source Catalogue, Astron. \& Astrophys. 536, A7 (2011).

[8] M. Juvela, I. Ristorcelli, L. A. Montier et al., Galactic cold cores: Herschel study if first Planck detections, Astron. \& Astrophys. 518, L93 (2010).

[9] M. Juvela, I. Ristorcelli, L. Pagani et al., Galactic cold cores III. General cloud properties, Astron. \& Astrophys. 541, A12 (2012).

[10] L. A. Wilcock et al., Cores in infrared dark clouds (IRDCs) seen in the Hi-GAL survey between $l=300$ degrees and 330 degrees, Mont. Not. Roy. Astron. Soc. 422, 1071 (2012).

[11] M. Veneziani, P. A. R. Ade, J. J. Bock et al., Properties of galactic cirrus clouds observed by BOOMERanG, Astrophys. J. 713, 959-969 (2010). 
[12] Th. M. Nieuwenhuizen, R. E. Schild and C. H. Gibson, Do micro brown dwarf detections explain the galactic dark matter?, J. Cosmol. 15, 6017-6029 (2011).

[13] SMC: http://www.nasa.gov/mission_pages/herschel/multimedia/pia15255.html.

[14] LMC: http://www.nasa.gov/mission_pages/herschel/multimedia/pia15254.html.

[15] J. H. Jeans, The Stability of a Spherical Nebula, Phil. Trans. Roy. Soc. London, A 199, 1 (1902).

[16] C. H. Gibson, Turbulence in the Ocean, Atmosphere, Galaxy, and Universe, Appl. Mech. Rev. 49, 299-315 (1996).

[17] Th. M. Nieuwenhuizen, Explanation of the Helium-3 problem, J. Cosmol. 15, 6200-6203 (2011).

[18] S. Kida, K. Niinuma, S. Suzuki et al., Two strong radio bursts at high and medium Galactic latitude, New Astronomy 13, 519-525 (2008).

[19] D. A. Frail, S. R. Surkarni, E. O. Ofek et al., A revised view of the transient radio sky, Astrophys. J. 747, 70 (2012).

[20] J. H. Oort, The formation of galaxies and the origin of the high-velocity hydrogen, Astron. \& Astrophys. 7, 381 - 404 (1970).

[21] R. B. Larson, Effect of infalling matter on the heavy element content of a galaxy, Nature 236, 7 (1972).

[22] K. H. R. Rubin, J. Xavier Prochaska, D. C. Koo and A. C. Phillip, The Direct Detection of Cool, Metal-Enriched Gas Accretion onto Galaxies at $z \sim 0.5$, Astrophys. J. 747, L26 (2012).

[23] M. Rauch, The Lyman Alpha Forest in the Spectra of QSOs, Ann. Rev. Astron. \& Astrophys. 36, 267-316 (1998).

[24] J. E. G. Peek, Carl Heiles, K. M. G. Peek, et al., The Local Leo Cold Cloud and New Limits on a Local Hot Bubble, Astrophys. J. 735, 129 (2011).

[25] D. M. Meyer, J. T. Lauroesch, J. E. G. Peek and C. Heiles, The remarkable pressure of the Local Leo Cold Cloud, Astrophys. J. 752, 119 (2012).

[26] R. E. Schild and C. H. Gibson, Primordial Planet Formation, J. Cosmol. 15, 6156-6183 (2011).

[27] C. H. Gibson and R. E. Schild, Formation of Planets by Hydrogravitational Dynamics, J. Cosmol. 15, 6184-6199 (2011).

[28] S. M. Fall, R. Chandar and B. Whitmore, The Age Distribution of Massive Star Clusters in the Antennae galaxies, Astrophys. J. 631, L133-136 (2005).

[29] H.D. Tran, M. Sirianni, H. C. Ford et al., Advanced Camera for Surveys Observations of Young Star Clusters in the Interacting Galaxy UGC 10214, Astrophys. J. 585, 750-755 (2003).

[30] T. H. Puzia, M. Kissler-Patig, Jean P. Brodie and John P. Huchra, The Age Difference between the Globular Cluster Sub-populations in NGC 4472, Astron. J. 118, 2734 (1999).

[31] X.-X. Xue, H.-W. Rix, G. Zhao et al., The Milky Way's Circular Velocity Curve to $60 \mathrm{kpc}$ and an Estimate of the Dark Matter Halo Mass from Kinematics of 2400 SDSS Blue Horizontal Branch Stars, Astrophys. J. 684, 1143-1158 (2008).

[32] M. Honma et al., to appear, (2012). http://www.nao.ac.jp/E/release/2012/10/03/mass-of-darkmatter-revealed-by-precise-measurements-of-the-galaxy.html

[33] M. J. Griffin, A. Abergel, A. Abrue et al., The Herschel-SPIRE instrument and its in-flight performance, Astron. \& Astrophys. 518, L3 (2010).

[34] M. Meixner, F. Galliano, S. Hony et al., Herschel Inventory of The Agents of Galaxy Evolution (HERITAGE): the Large Magellanic Cloud dust, Astron. \& Astrophys. 518, L71 (2010).

[35] J. Kainulainen, J. Alves, H. Beuther, T. Henning and F. Schuller, Mass reservoirs surrounding massive infrared dark clouds: A view by near-infrared dust extinction, Astron. Astrophys. 536, A48 (2011).

[36] Th. M. Nieuwenhuizen, C. H. Gibson and R. E. Schild, Gravitational hydrodynamics of large-scale structure formation, Europhys. Lett. 88, 49001 (2009).

[37] R. E. Schild, Microlensing variability of the gravitationally lensed quasar Q0957+561 A,B, Astrophys. J. 464, 125 (1996).

[38] R. E. Schild and M. Dekker, The Transparency of the Universe Limited by Ly-alpha Clouds, Astronom. Nachr. 327, 729-732 (2006). 
[39] E. F. G. van Heusden and M. P. Liska, "On dark matter in the Milky Way and the Magellanic Clouds", oral presentation, University of Amsterdam (June 29, 2012).

[40] W. E. Harris, A catalog of parameters for globular clusters in the Milky Way, Astron. J. 112, 1487 (1996). See also the database at http://physwww.mcmaster.ca/ harris/mwgc.dat.

[41] C. Renault, E. Aubourg, P. Bareyre, et al., Search for planetary mass objects in the Galactic halo through microlensing. Astron. \& Astroph. 329, 522 (1998).

[42] C. Alcock, R. A. Allsman, D. Alves et al., The MACHO Project: Microlensing Results from 5.7 Years of LMC Observations, Astrophys. J. 542, 281 (2000).

[43] E. Rivers, A. Markowitz and R. Rothschild, An Occultation Event in Centaurus A and the Clumpy Torus Model, Astrophys. J. 742, L29 (2011).

[44] W. N. Colley and R. E. Schild, A rapid microlensing event in the Q0956+561A, B gravitational lens system, Astrophys. J. 594, 97 (2003).

[45] Th. M. Nieuwenhuizen, A partially occulting MACHO-microlensing event in the Twin Quasar Q0957+261 A,B, to appear.

[46] Th. M. Nieuwenhuizen, Do non-relativistic neutrinos constitute the dark matter? Europhys. Lett. 86, 59001 (2009).

[47] Y. Y. Y. Wong, Neutrino mass in cosmology: status and prospects, Ann. Rev. Nucl. Part. Sci. 61, 69 (2011). 